\title{
F-Box Family Genes, LTSF1 and LTSF2, Regulate Low-Temperature Stress Tolerance in Pepper (Capsicum chinense)
}

\author{
Jelli Venkatesh ${ }^{1}{ }^{\circledR}$, Min-Young Kang ${ }^{1}$, Li Liu ${ }^{2}$, Jin-Kyung Kwon ${ }^{1}$ and Byoung-Cheorl Kang ${ }^{1, *}$ \\ 1 Department of Agriculture, Forestry and Bioresources, Research Institute of Agriculture and Life Sciences, \\ Plant Genomics and Breeding Institute, College of Agriculture and Life Sciences, Seoul National University, \\ Seoul 08826, Korea; jvs15@snu.ac.kr (J.V.); kmyjj3802@korea.kr (M.-Y.K.); kwonjk@snu.ac.kr (J.-K.K.) \\ 2 Global Institute for Food Security, University of Saskatchewan, Saskatoon, SK S7N 5A8, Canada; \\ li.liu-green@gifs.ca \\ * Correspondence: bk54@snu.ac.kr; Tel.: +82-2-880-4563; Fax: +82-2-873-2056
}

Received: 7 August 2020; Accepted: 8 September 2020; Published: 11 September 2020

\begin{abstract}
The F-box proteins belong to a family of regulatory proteins that play key roles in the proteasomal degradation of other proteins. Plant F-box proteins are functionally diverse, and the precise roles of many such proteins in growth and development are not known. Previously, two low-temperature-sensitive F-box protein family genes (LTSF1 and LTSF2) were identified as candidates responsible for the sensitivity to low temperatures in the pepper (Capsicum chinense) cultivar 'sy-2'. In the present study, we showed that the virus-induced gene silencing of these genes stunted plant growth and caused abnormal leaf development under low-temperature conditions, similar to what was observed in the low-temperature-sensitive ' $s y$-2' line. Protein-protein interaction analyses revealed that the LTSF1 and LTSF2 proteins interacted with S-phase kinase-associated protein 1 (SKP1), part of the Skp, Cullin, F-box-containing (SCF) complex that catalyzes the ubiquitination of proteins for degradation, suggesting a role for LTSF1 and LTSF2 in protein degradation. Furthermore, transgenic Nicotiana benthamiana plants overexpressing the pepper LTSF1 gene showed an increased tolerance to low-temperature stress and a higher expression of the genes encoding antioxidant enzymes. Taken together, these results suggest that the LTSF1 and LTSF2 F-box proteins are a functional component of the SCF complex and may positively regulate low-temperature stress tolerance by activating antioxidant-enzyme activities.
\end{abstract}

Keywords: F-box protein; low-temperature stress; proteasomal degradation; transgenics; virus-induced gene silencing; SCF complex

\section{Introduction}

In plants, low temperatures affect many physiological processes, including water and nutrient uptake, photosynthesis, immune responses, growth, and development, as well as the geographical distribution of plants [1,2]. Enhancing the low-temperature tolerance of temperature-sensitive crops is necessary to increase their productivity [3-5]. Pepper, an important fruit crop grown worldwide, is temperature-sensitive, with an optimum temperature of $25-30{ }^{\circ} \mathrm{C}[6,7]$. Deviations from these temperatures can adversely affect growth and development, resulting in a variety of developmental and physiological disorders [6-8].

Many plant species overcome the adverse effects of low and non-freezing temperatures through complex adaptive mechanisms which are associated with the extensive reprogramming of the expression of a number of cold-responsive genes and subsequent changes in numerous physiological and biochemical processes, such as membrane stability, calcium ion fluxes, and changes in the properties of 
the cell wall and the plasma membrane [9-12]. To elucidate the molecular basis of low-temperature sensitivity in plants, extensive studies have been performed using low-temperature-sensitive mutants. The Arabidopsis thaliana fatty acid desaturase 2 (fad2) mutant, which is deficient in the endoplasmic reticulum 18:1 desaturase, displays abnormal leaves and a dwarf phenotype under low temperatures [13,14]. The Arabidopsis nonphotochemical quenching 1 (npq1) mutant, defective in xanthophyll biosynthesis, excessively accumulates reactive oxygen species (ROS) [15]. The Arabidopsis BONZAI 1 (BON1) and $B O N 1$-associated protein (BAP1) genes were suggested to function directly in the regulation of cell expansion and cell division at lower temperatures, although the miniature bon 1 mutant plants were fertile [16]. The maize (Zea mays) mutant inbred line M11 and the rice (Oryza sativa) low temperature albino 1 (lta1) mutants contained remarkably low levels of chlorophyll $[17,18]$. Despite these insights, little is known about the genes and mechanisms regulating low-temperature tolerance in plants.

The pepper cultivar ' $s y-2$ ' is a temperature-sensitive landrace local to the Seychelles, and shows developmental defects, such as abnormal leaf growth, chlorophyll deficiency, the excessive accumulation of ROS, and altered fatty acid biosynthesis, when grown at temperatures lower than $24{ }^{\circ} \mathrm{C}[6,8,19]$. Using genetic mapping, two low-temperature-sensitive F-box (LTSF) proteins, LTSF1 and LTSF2 (encoded by ORF10 and ORF20, respectively), belonging to the Skp, Cullin, F-box-containing (SCF) complex of ubiquitin-proteasome machinery, were identified as candidates responsible for the low-temperature sensitivity in the pepper cultivar ' $s y-2$ ' [19]; however, the functional relevance of the LTSF1 and LTSF2 genes in the temperature sensitivity of the 'sy-2' cultivar is still unclear.

The ubiquitin-proteasome pathway plays a central regulatory role in the selective degradation of target proteins through a series of steps. First, the ubiquitin is activated by the E1 enzyme (ubiquitin-activating enzyme), then the activated ubiquitin is transferred to E2 (ubiquitin-conjugating enzyme). The E2 transfers ubiquitin to the E3 (ubiquitin protein ligase) after which the ubiquitin is transferred to the target protein by the E3 complex [20]. F-box proteins are constituent subunits of the multi-protein E3 ubiquitin ligase (also known as the SCF complex) and confer target specificity during the $26 \mathrm{~S}$ proteasome-mediated protein degradation [20]. The F-box family is one of the largest multigene families in plants [21]. A large number of F-box proteins have been identified in searches for their highly-conserved N-terminal F-box domain, and a total of 694, 687, 337, and 156 F-box family genes have been reported in Arabidopsis, rice, poplar, and grape, respectively [22,23]. F-box protein families can be grouped into several subfamilies based on their C-terminal target-specific protein-protein interaction domains, such as the leucine-rich repeats (LRRs), tubby (TUB), tetratricopeptide (TPR), kelch repeats, Trp-Asp forty-amino-acid repeat (WD-40), armadillo repeats (ARMs), and RING finger domains [24-26]. The presence of numerous diverse F-box proteins facilitates the formation of diverse SCF complexes that can recognize a broad array of substrates, and can, thus, be associated with the regulation of numerous biophysiological processes, such as plant growth and development, hormonal responses, biotic and abiotic stress responses, and senescence [21,27].

In this study, we performed a functional characterization of the low-temperature sensitive pepper F-box protein genes LTSF1 and LTSF2 using virus-induced gene silencing (VIGS) and transgenic approaches. The silencing of LTSF1 and LTSF2 expression in the low-temperature-tolerant pepper line 'No.3341' resulted in a low-temperature-sensitive phenotype similar to 'sy-2'. Transgenic tobacco (Nicotiana benthamiana) lines overexpressing the pepper LTSF1 allele from 'No.3341' displayed an enhanced tolerance to low temperatures relative to the control.

\section{Results}

\subsection{LTSF1 and LTSF2 Sequence Analysis}

We characterized the LTSF1 and LTSF2 genes and proteins believed to be affected in the 'sy-2' pepper mutant [19]. The predicted LTSF1 and LTSF2 proteins consisted of 450 and 467 amino acids (AAs), respectively (Supplementary Materials Figure S1), with predicted molecular weights of about 51.8 and isoelectric points of 9.31. A search using the InterPro protein sequence analysis and 
classification software tool identified two conserved domains in both LTSF1 and LTSF2: an F-box domain (IPR001810) in the N-terminus and an F-box-associated (FBA) interaction motif (IPR017451) in the C-terminus (Figure 1). The presence of the F-box domain indicated that LTSF1 and LTSF2 are F-box proteins, which bind to S-phase kinase-associated protein 1 (SKP1) to form the SCF complex. FBA motifs are generally involved in interactions with the target proteins to be ubiquitinated for proteasomal degradation.

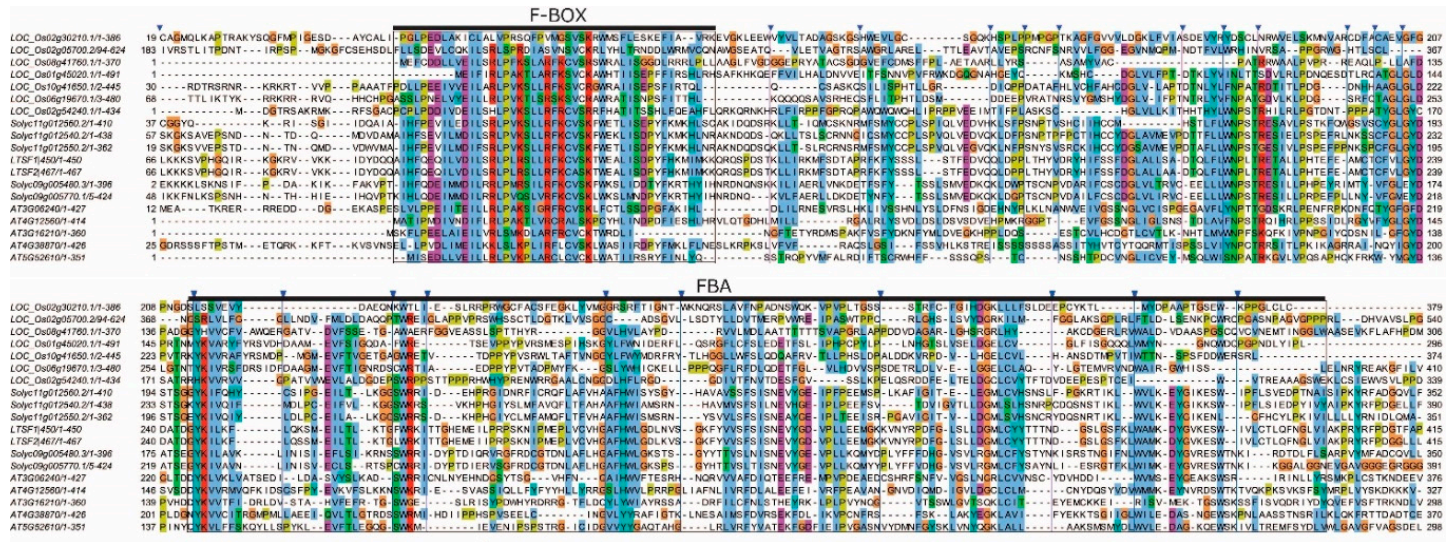

Figure 1. Multiple-sequence alignments of two low-temperature-sensitive F-box (LTSF) proteins, LTSF1 and LTSF2, in pepper ('No.3341') with their homologs in Arabidopsis, tomato, and rice. The predicted F-box and F-box-associated (FBA) domains are indicated above the alignment. Amino acid (AA) residues are colored according to ClustalX color code using Jalview tool.

The LTSF1 and LTSF2 proteins share a 91\% sequence similarity, with a single AA difference in their F-box domains. The protein sequence alignment of the F-box domains of LTSF1 and LTSF2 in pepper and other plant species, including Arabidopsis, rice, and tomato, revealed a high sequence conservation in the F-box domain (Figure 1). LTSF1 and LTSF2 showed a maximum sequence identity of $41-42 \%$ with the tomato F-box protein Solyc09g005480, and shared a sequence identity of 24-25\% with the FBA motif-containing Arabidopsis At4g12560 protein (CPR1; CONSTITUTIVE EXPRESSER OF PR GENES 1); At3g06240 (a F-box/kelch-repeat protein); and rice Os02g54240, a stress-responsive F-box protein. A comparison of the genomic structures revealed that both the LTSF1 and LTSF2 genes contain two introns (Figure 2A).

\subsection{VIGS Analysis}

To test the functional roles of LTSF1 and LTSF2 in low-temperature sensitivity, we silenced their expression in wild-type pepper ('No.3341') using tobacco rattle virus (TRV)-mediated VIGS (Figure 2A,B). VIGS vectors were constructed targeting the UTR or coding (CDS) regions. To confirm the efficient silencing of the genes, a qPCR was performed in the silenced plants. No significant changes were observed in the expression levels of the LTSF1 and LTSF2 genes in plants infiltrated with constructs targeting either LTSF1 or LTSF2 gene UTR regions due to the unsuccessful gene silencing, possibly due to shorter VIGS target region of $135 \mathrm{bp}$ and $127 \mathrm{bp}$, respectively for LTSF1 and LTSF2. However, in plants infiltrated with VIGS construct (LTSF1/2-double-silenced plants) targeting CDS regions of both LTSF1 and LTSF2 with 305 bp target length (Figure 2A), the expression levels of these genes were approximately four times lower compared with the mock plants (Figure 2C). The double silencing of the LTSF1 and LTSF2 genes by targeting their CDS region resulted in an abnormal phenotype and severe growth retardation under low-temperature conditions $\left(20^{\circ} \mathrm{C}\right)$, consistent with the suppressed expression of LTSF1 and LTSF2 in these plants (Figure 2B,C). The LTSF1/2-silenced plants produced small, irregularly shaped, pale, and thick leaves, similar to the phenotype observed in the 'sy-2' pepper mutant, which exhibits thick irregularly shaped leaves when grown under low-temperature 
conditions [19]. Plant height and leaf area were markedly affected in the LTSF1/2-silenced plants as compared with mock plants infiltrated with empty vector (TRV2:00). These results indicate an important role for the F-box genes in low-temperature sensitivity and morphogenesis in the pepper plants.

A

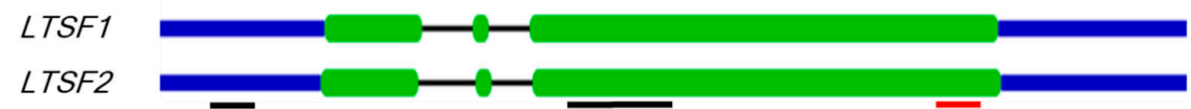

B

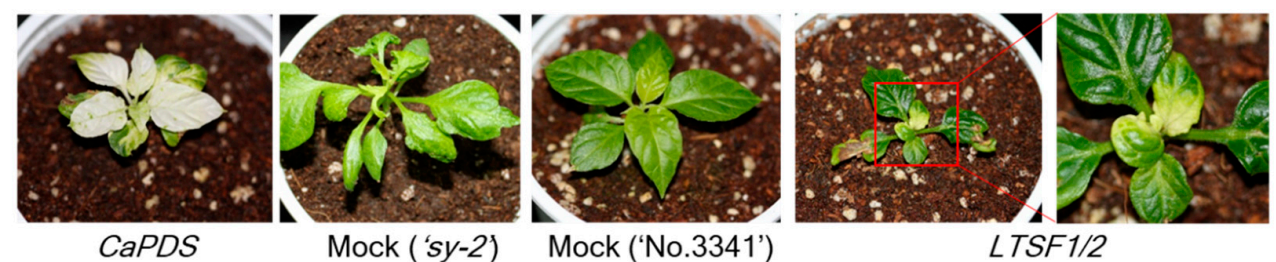

C

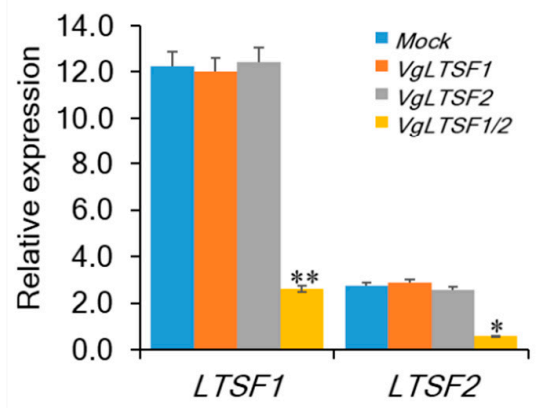

D

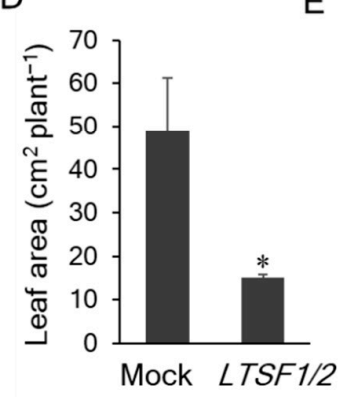

$E$

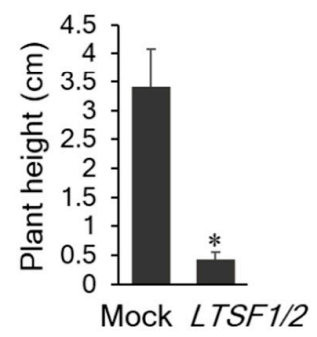

Figure 2. Virus-induced gene silencing (VIGS) of the pepper LTSF1 and LTSF2 genes in wild-type ('No.3341') plants. (A) Schematic structure of the LTSF1 and LTSF2 gene sequences. Closed green boxes and solid black lines indicate exons and introns, respectively. Blue lines indicate untranslated regions (UTRs). The VIGS target sites in LTSF1 and LTSF2 are underlined with black lines and qPCR target region is underlined with red line. (B) VIGS pepper phenotypes. Pepper plants with VIGS of Capsicum phytoene desaturase (CAPDS) showed a photobleaching phenotype. The double silencing of the LTSF1/2 genes resulted in plants with an abnormal phenotype. The rightmost image is a magnification of the area highlighted by a red square. (C) Quantitative PCR (qPCR) analysis of plants in which LTSF1 and/or LTSF2 were silenced using VIGS $(V g)$. VgLTSF1 and $V g L T S F 2$ refer to the VIGS constructs targeting the UTRs. (D) Quantitative analysis of leaf area in mock ('No.3341') and LTSF1/2 silenced plants. (E) Quantitative analysis of plant height in mock ('No.3341') and LTSF1/2 silenced plants. The error bars indicate the mean \pm standard deviation (SD) of six biological replicates. ${ }^{*} p<0.05$ and ${ }^{* *} p<0.01$ vs. control (mock).

\subsection{LTSF1 and LTSF2 Interaction with SKP1}

LTSF1 and LTSF2 were predicted to encode F-box proteins containing a C-terminal FBA domain (Figure 1). To determine whether the LTSF1 and LTSF2 proteins are functional components of the SCF complex, protein-protein interaction studies were carried out (Figure 3A). SKP1 interacts with F-box proteins by binding to their conserved F-box domain [20]; therefore, we performed yeast two-hybrid (Y2H) assays to assess whether pepper SKP1 can interact with the Sy-2 candidates LTSF1 and LTSF2. No significant growth was detected in yeast cells carrying the control vectors on the selection medium (SD/-Leu/-Trp/-His/3-AT); however, the yeast cells carrying the pGAD-T7 vector containing the F-box domain sequence of LTSF1 or LTSF2 (LTSF1/2-F2) and pGBK-T7:SKP1 grew well (Figure 3A), suggesting that LTSF1/LTSF2 can interact with SKP1. Consistent with these results, $\beta$-galactosidase (GUS) assay raveled a strong interaction between LTSF1/LTSF2 and SKP1 (Figure 3B). The growth of the yeast cells carrying the pGAD-T7 vector with the full-length sequence of LTSF1 or LTSF2 (LTSF1/2-F1) and the pGBK-T7:SKP1 was inhibited on the SD plate, however (Figure 3A). This weak or absent interaction 
between the full-length LTSF1/LTSF2 proteins and SKP1 may suggest that the C-terminal FBA domains may have an inhibitory effect in yeast cells. Alternatively, it is possible that the low effectivity of interaction could also be caused by different expression levels of the proteins.

A
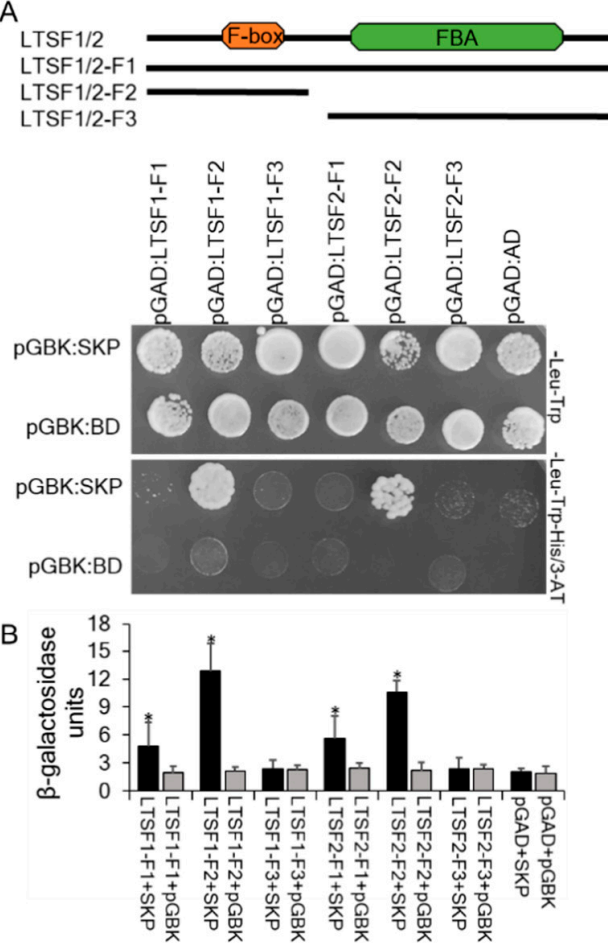

C

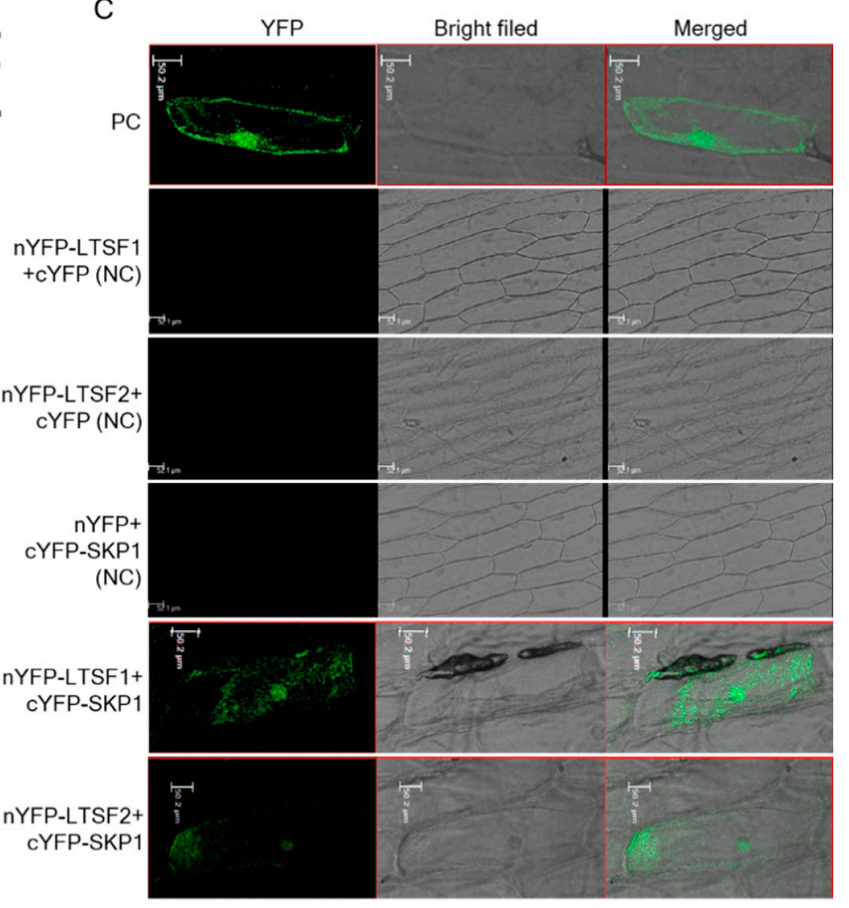

Figure 3. Protein-protein interaction studies involving LTSF1 and LTSF2. (A) Yeast two-hybrid assays analyzing the interactions between LTSF1/2 and SKP1. The LTSF1, LTSF2, and SKP1 coding sequences were cloned into the pGAD-T7 and pGBK-T7 vectors. Yeast AH109 cells transformed with the designated plasmid combinations were grown on SD/-Leu/-Trp and SD/-Leu/-Trp/-His/+3-aminotriazol (3-AT) media. (B) $\beta$-galactosidase (GUS) activity assay. Error bars indicate SD of five biological replicates of relative GUS activity assay. The asterisk indicates a significant difference compared with the negative construct $(p<0.05)$. (C) Bimolecular fluorescence complementation assay of the interactions between pepper LTSF1/2 and SKP1. The LTSF1, LTSF2, and SKP1 coding sequences were cloned into the pSPYCE and pSPYNE vectors. The designated plasmid combinations were co-bombarded into onion epidermal cells for the transient expression of yellow fluorescent protein (YFP), and the fluorescence signals were visualized using a laser-scanning confocal microscope. PC and NC indicate positive and negative controls.

To confirm the protein-protein interaction between LTSF1/LTSF2 and SKP1 in plant cells, a bimolecular fluorescence complementation (BiFC) assay was performed. LTSF1 and LTSF2 were fused with the C-terminus of YFP, and SKP1 was fused with the N-terminus of YFP. These constructs were then co-bombarded into onion epidermal cells. The negative control failed to produce a BiFC signal, while a strong fluorescent signal was observed throughout the cells transformed with the positive controls. A strong fluorescent signal was observed in the nuclei, cytoplasm, and cell membranes of epidermal cells co-bombarded with the YFP fusion proteins of LTSF1 and SKP1 (Figure 3C), indicating a strong interaction between LTSF1 and SKP1, whereas a weak fluorescent signal was observed in the epidermal cells co-bombarded with the YFP fusion proteins of LTSF2 and SKP1, indicating a weak interaction between LTSF2 and SKP1 (Figure 3C). Overall, these results indicate that both LTSF1 and LTSF2 interact with SKP1 in plant cells, and the detection of the BiFC signals in the nucleus, cytoplasm, and plasma membrane indicates that LTSF1 and LTSF2 may be involved in proteasome degradation in both the nucleus and the cytoplasm. 


\subsection{The Transgenic Expression of LTSF1 Promotes Low-Temperature Stress Tolerance in N. benthamiana Plants}

To confirm the role of the pepper F-box gene LTSF1 in low-temperature stress tolerance, transgenic $N$. benthamiana lines overexpressing LTSF1 were developed (Figure 4A,B). The genotypes of the $\mathrm{T}_{0}$ transgenic LTSF1-expressing lines were confirmed using reverse-transcription PCR (RT-PCR) analysis (Figure S2). Two transgenic lines (LTSF1-TG1 and -TG2) and their corresponding vector control (VC) plants were grown under normal $\left(25^{\circ} \mathrm{C}\right)$ and low $\left(14^{\circ} \mathrm{C}\right)$ temperatures for one month. No significant morphological changes were observed between the LTSF1-overexpressing $N$. benthamiana lines and the control plants under the normal conditions; however, the reduced plant growth and shorter heights induced in the control plants by the low-temperature conditions were less severe in the transgenic lines, which grew approximately 1.5 times taller than the control plants (Figure 4B,C). Thus, the transgenic expression of LTSF1 alleviated the effect of low-temperature stress in the LTSF1-TG N. benthamiana plants.
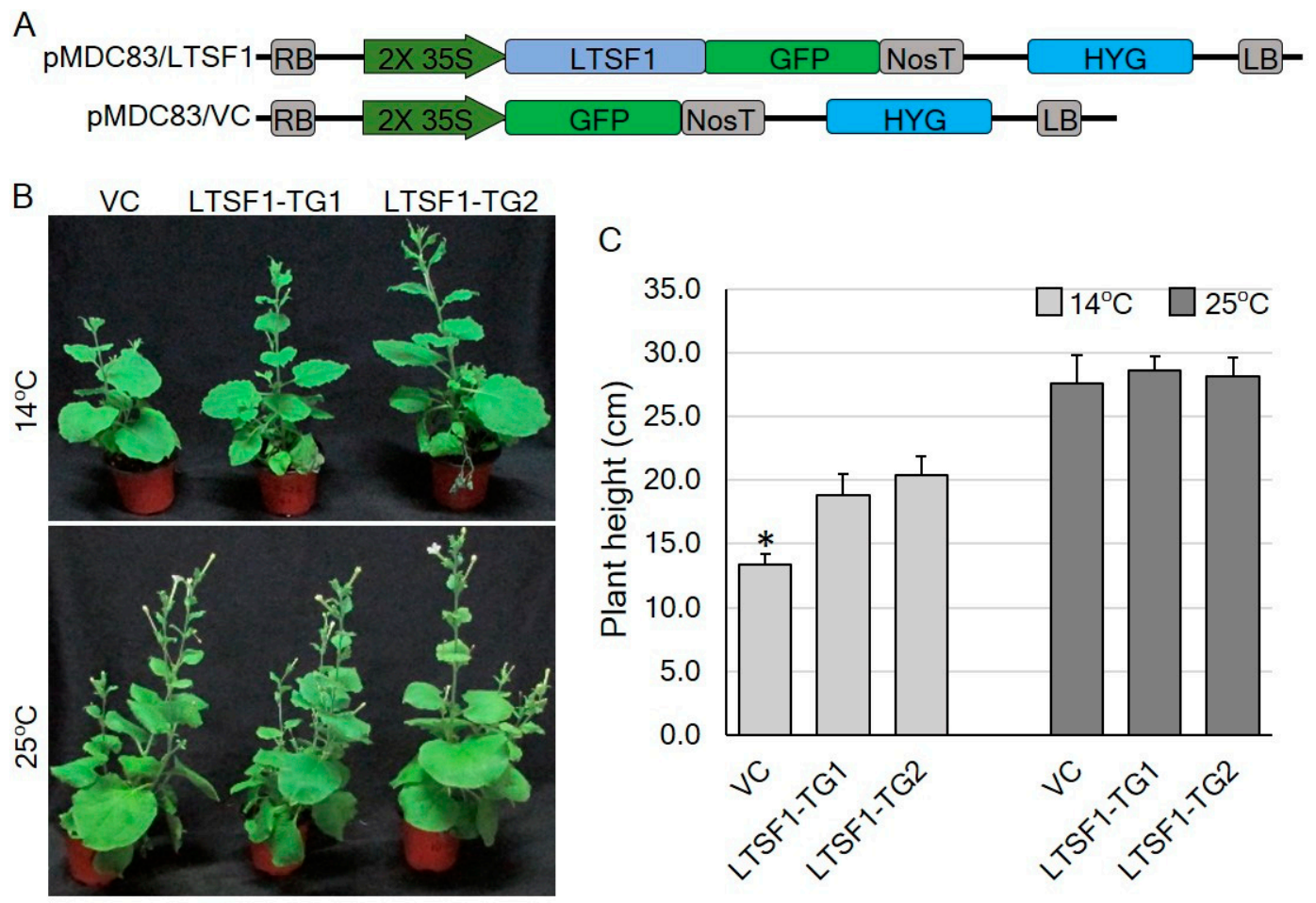

Figure 4. Transgenic N. benthamiana plants overexpressing LTSF1. (A) Schematic diagram of the transfer DNA (T-DNA) region of the pMDC83/VC and pMDC83/LTSF1 vectors: RB, right border of T-DNA region; LB, left border of T-DNA region; $2 \times 35$, Cauliflower Mosaic Virus 35 S promoter; HYG, hygromycin phosphotransferase gene; NosT, nopaline synthase terminator. (B) Phenotype of the LTSF1-overexpressing and vector control (VC) transgenic N. benthamiana plants after 30 days of low-temperature stress $\left(14^{\circ} \mathrm{C}\right)$. (C) Plant heights of the LTSF1-overexpressing transgenic and VC plants under temperature stress $\left(14^{\circ} \mathrm{C}\right)$ and normal conditions $\left(25^{\circ} \mathrm{C}\right)$. The error bars indicate the mean $\pm \mathrm{SD}$ of six biological replicates. ${ }^{*} p<0.01$ vs. control (VC).

To determine whether LTSF1 regulates low-temperature stress through the antioxidant pathway, we analyzed the transcript abundances of the genes encoding ROS-scavenging antioxidant enzymes, such as ascorbate peroxidase (APX), catalase (CAT), glutathione S-transferase (GST), and superoxide dismutase (SOD), in the control and transgenic plants subjected to low-temperature stress. We observed a significant increase in the accumulation of APX transcripts in the transgenic LTSF1-TG1 and -TG2 plants compared with the control plants both under the stressed and non-stressed conditions (Figure 5). Similarly, the expression of GST and CAT was also significantly increased in the LTSF1-TG1 and -TG2 
plants under the stress conditions. The accumulation of SOD transcripts was higher in the LTSF1-TG1 and -TG2 transgenic plants subjected to the stress conditions than in those grown in the non-stressed conditions. Overall, the expression levels of the genes encoding the ROS-scavenging enzymes were considerably altered by the low-temperature stress, and their levels were higher in the transgenic plants than in the control plants. These results indicate that the transgenic expression of LTSF1 may influence the antioxidant system and, thus, confers low-temperature tolerance.
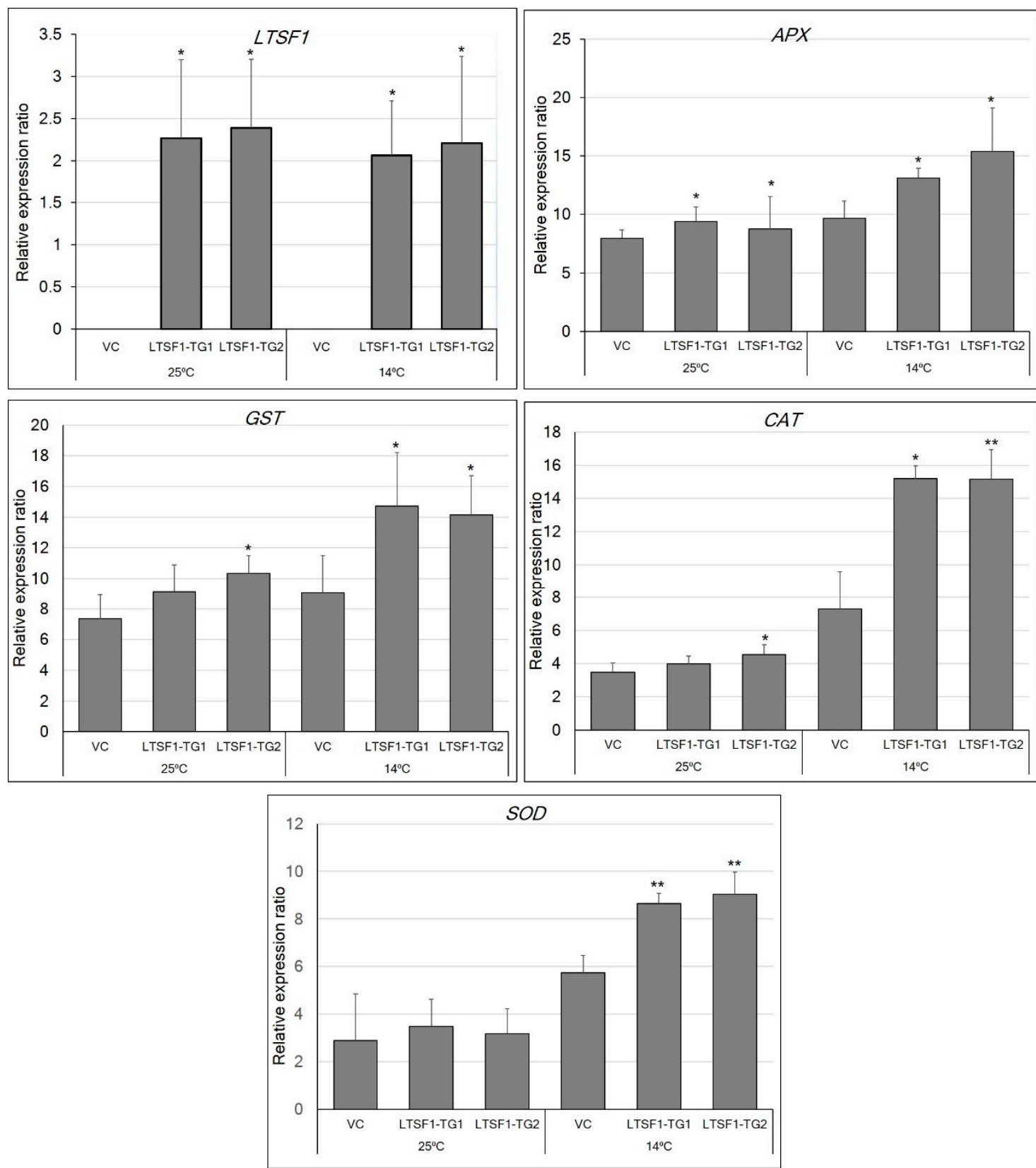

Figure 5. Relative expressions of levels of LTSF1 and antioxidant-enzyme related genes (ascorbate peroxidase (APX), catalase (CAT), glutathione S-transferases (GST), and superoxide dismutase (SOD)) in transgenic $N$. benthamiana plants. The error bars indicate the mean $\pm \mathrm{SD}$ of six biological replicates. ${ }^{*} p<0.05$ and ${ }^{* *} p<0.01$ vs. the vector control (VC). 


\section{Discussion}

The SCF complex is an E3 ubiquitin ligase that mediates the ubiquitination of proteins destined for proteasomal degradation in several plant developmental processes, including hormone signaling, morphogenesis, embryo development, circadian rhythms, and senescence [26]. F-box proteins are adaptor subunits of the SCF complex; the N-terminal F-box domain and the SKP subunit protein physically interact to form a core complex and recruit target proteins for proteasomal degradation through the C-terminal protein-protein interaction domain of the F-box protein [28-30].

In this study, the low-temperature stress-responsive LTSF1 and LTSF2 genes were predicted to encode F-box proteins containing a C-terminal FBA domain, which participates in protein-protein interactions associated with a wide range of plant physiological processes [31-34]. We cloned the F-box genes LTSF1 and LTSF2 from pepper and performed BiFC and Y2H assays to verify that LTSF1 and LTSF2 are functional F-box proteins. Protein-protein interaction studies revealed a weak interaction between LTSF2 and SKP1 compared with the LTSF1 and SKP1. The differences interaction between these proteins could be due to a single AA change (valine (V) in LTSF1 to alanine (A) in LTSF2) in their F-box domain (Figure 1).

To examine the subcellular localization of LTSF1 and LTSF2, we fused the LTSF1 and LTSF2 genes to the N-terminus of a green fluorescent protein (GFP) reporter gene and transformed the fusion protein expression vectors into $N$. benthamiana leaves for the transient expression of LTSF1/LTSF2:GFP fusion proteins. As shown in Figure S3, the green fluorescence signal was distributed throughout the cells infiltrated with the 35S::GFP control vector (Figure S3). Similarly, the fluorescence signal of the 35S::LTSF1/2-GFP fusion was detected throughout the cells, including in the nucleus, cytoplasm, and plasma membrane (Figure S3). In previous studies, SCF complex proteins, such as the tomato E3 ubiquitin ligase SIBAH1, the Arabidopsis SKP1-like protein13 (AtASK13), the wheat (Triticum aestivum) F-box protein TaFBA1, and the rice F-box protein OsMAIF1, were found to be involved in the tolerance of biotic and abiotic stresses, and were also documented to be localized to the nucleus, cytoplasm, and plasma membrane [35-38]. The dispersed subcellular localizations of LTSF1, LTSF2, and other SCF complex proteins may, therefore, contribute to their diverse roles in plant cells.

Various components of SCF complexes have been reported to be associated with plant growth, development, and the responses to biotic and abiotic stresses $[19,34-36,39,40]$ while a number of stress conditions have been documented to activate the expression of genes encoding the subunits of the SCF complex [28,41]. In the present study, to confirm the functional role of LTSF1 and LTSF2 genes in conveying low-temperature tolerance, we silenced the expression of these genes using VIGS. Silencing of the LTSF1 or LTSF2 gene by targeting their UTR region was not successful in our case. The double silencing of both LTSF1 and LTSF2 genes by targeting their CDS region resulted in an abnormal phenotype and severe growth retardation under low-temperature conditions $\left(20^{\circ} \mathrm{C}\right)$. Consistent with the suppressed expression of LTSF1/LTSF2, these double-silenced plants produced small, irregularly shaped, thick, and pale leaves (Figure 2), similar to those observed in the low-temperature-sensitive 'sy-2' cultivar grown under low temperatures [19]. Furthermore, VIGS of the LRR domain-containing Capsicum annuum F-box (CaF-box) gene reduced the cold-stress tolerance of pepper seedlings [42] supporting the role of the F-box genes in regulating the temperature sensitivity of plants.

To further confirm the role of LTSF1 and LTSF2 in low-temperature stress, we developed LTSF1 overexpressing transgenic $N$. benthamiana lines. We observed that the growth of the vector control plants in terms of their height was more severely inhibited by low-temperature stress than in the transgenic lines overexpressing LTSF1. The transgenic plants showed enhanced transcriptional activity for the genes encoding the antioxidant enzymes, including SOD, APX, CAT, and GST (Figure 5), which could be associated with the improved temperature tolerance shown by the transgenic plants. Similar results were observed in transgenic wheat and tobacco plants overexpressing the TaFBA1 F-box gene, in which increased antioxidant-enzyme activity was correlated with increased abiotic stress $[37,43]$. In another study, the overexpression of SKP1 improved the resistance of Arabidopsis to various abiotic stresses, including heat, salinity, and oxidative stress [35], whereas the T-DNA insertion 
mutants and RNAi mutant lines lacking the related ASK13 gene showed a low tolerance to abiotic stress [35]. Our previous study revealed that the cell death and leaf deformation observed in the 'sy-2' mutant grown under low temperatures is caused by the excessive accumulation of ROS, and is correlated with the downregulation of the genes encoding ROS-scavenging enzymes at $20{ }^{\circ} \mathrm{C}$ [19]. In the present study, N. benthamiana plants overexpressing LTSF1 showed a markedly increased expression of ROS-scavenging enzyme-encoding genes, such APX, CAT, GST, and SOD, further substantiating the role of the ROS-scavenging pathway in low-temperature stress tolerance (Figure 5 ). The F-box protein LTSF1, which is a key subunit of the SCF complex, may, therefore, play an important role in positively regulating low-temperature tolerance by modulating the stress-related genes, possibly through the repression of the negative regulators of cold stress.

A previous study revealed that the cell death and leaf deformation observed in the ' $s y-2$ ' mutant grown under low temperatures is caused by the excessive accumulation of ROS, and is correlated with the downregulation of the genes encoding ROS-scavenging enzymes, such as APX6, APXT1, and GST1, at $20{ }^{\circ} \mathrm{C}$ [6]. Furthermore, salt-tolerant transgenic tobacco plants overexpressing the TaFBA1 gene showed increased antioxidant-enzyme activity along with increased E3 ligase enzyme activity [44]. In the present study, N. benthamiana plants overexpressing LTSF1 showed a markedly increased expression of ROS-scavenging enzyme-encoding genes, such APX, CAT, GST, and SOD, further substantiating the role of the ROS-scavenging pathway in low-temperature stress tolerance (Figure 5). However, further experiments will be needed to determine whether the increased transcriptional activity of antioxidant-enzyme genes in transgenic N. benthamiana plants overexpressing LTSF1 could be associated with increased E3 ligase activity.

\section{Materials and Methods}

\subsection{Plant Materials}

For the functional characterization of the two F-box candidate genes using VIGS, the pepper (C. chinense) 'No.3341' (wild-type) and 'sy-2' (mutant) cultivars [19] were used. For the functional studies of the F-box genes using heterologous expression, tobacco (N. benthamiana) was used. Plants were maintained under a $16 / 8 \mathrm{~h}$ light-dark regime at $24 \pm 2{ }^{\circ} \mathrm{C}$.

\subsection{Phylogenetic Analysis and Identification of Conserved Motifs}

Homologs of LTSF1 and LTSF2 (Table S1) were identified using a Basic Local Alignment Search tool (BLAST) search of other plant genomes in the National Center for Biotechnology Information (NCBI) database with default parameters using the LTSF1 and LTSF2 protein sequences as queries. To verify the conserved domains, the F-box homologous protein sequences were compared with known sequences using the ClustalW2 alignment tool with default parameters [45]. A multiple-sequence alignment of the LTSF1 and LTSF2 proteins and their homologs from other plant species, including Arabidopsis, tomato (Solanum lycopersicum), and rice, was created using the ClustalW2 program with default parameters [28]. The InterPro protein sequence analysis and classification tool [46] was used for the identification of the functional domains.

\subsection{VIGS}

Total RNA was extracted from the pepper ('No.3341') leaf samples using an MG RNAzol kit (MGmed, Seoul, Korea), according to the manufacturer's instructions. Complementary DNA (cDNA) was synthesized from $1 \mu \mathrm{g}$ RNA using EasyScript Reverse Transcriptase kit (TransGen Biotech, Beijing, China). VIGS target sequences in the LTSF1 and LTSF2 genes were amplified from the cDNA sequences of pepper using Pfu DNA Taq polymerase (Bioneer, Seoul, Korea) and gene-specific primers (Table 1). The target sequence for silencing of LTSF1 and LTSF2 genes were predicted by sol genomics VIGS tool [47] via BLAST against the Capsicum annuum v1.55 genome. Ligation-independent cloning (LIC) was used to clone the VIGS target sequences (LTSF1 (135 bp), LTSF2 (127 bp), and LTSF1/2 
(305 bp)) into the TRV VIGS vector (TRV2), as previously described [48]. Plasmids from the positively transformed clones were isolated, purified, and sequenced at the National Instrumentation Center for Environmental Management (NICEM; Seoul National University, Seoul, Korea). The Agrobacterium tumefaciens strain GV3101 was transformed with the pTRV2 (containing the target sequences) and pTRV1 vectors, then used for the coinfiltration of 3-week-old pepper cotyledons using a needleless syringe, as previously described [48]. Control plants infiltrated with empty vector (TRV2:00) and TRV1 were used as mock. Three weeks after coinfiltration of TRV constructs, quantitative measurements including leaf area and plant height were recorded. The VIGS experiment was repeated two times with least three biological replicates in each trail. ImageJ software was used for the analysis of leaf area.

Table 1. Cloning primers used in this study.

\begin{tabular}{|c|c|c|}
\hline Primer Name & Sequence & Purpose \\
\hline Y2H: LTSF1/2: NdeF1 & GAGACATATGATGCCTGTCAAAGTAGCA & $\mathrm{Y} 2 \mathrm{H}$ vector cloning \\
\hline Y2H: LTSF1/2: BamR1 & GAGAGGATCCTTAGATAACTAATTTTGGAGA & $\mathrm{Y} 2 \mathrm{H}$ vector cloning \\
\hline Y2H: LTSF1/2: NdeF2 & GAGACATATGATAGATTATGATCAGCAGGCAAT & $\mathrm{Y} 2 \mathrm{H}$ vector cloning \\
\hline Y2H: LTSF1/2: BamR2 & GAGAGGATCCCTATACATCCTCAAAAGTGGATA & $\mathrm{Y} 2 \mathrm{H}$ vector cloning \\
\hline Y2H: LTSF1/2: NdeF3 & TATACATATGGTACAACAACTCGATCCCCCT & $\mathrm{Y} 2 \mathrm{H}$ vector cloning \\
\hline SKP1-F-EcoRI & TCGCGAATTCATGTCTGCCCCAAAGAAAAT & $\mathrm{Y} 2 \mathrm{H}$ vector cloning \\
\hline SKP1-R-BamHI & TATAGGATCCTCACTCAAAGGCCCAAGCAT & $\mathrm{Y} 2 \mathrm{H}$ vector cloning \\
\hline SKP1:F-BamHI & CAATGGATCCATGTCTGCCCCAAAGAAAAT & BiFC vector cloning \\
\hline SKP1:R-XhoI & ATATCTCGAGCTCAAAGGCCCAAGCA & BiFC vector cloning \\
\hline LTSF1/2-F-BamHI & ACAGGGATCCATGCCTGTCAAAGTAGCA & BiFC vector cloning \\
\hline LTSF1/2-R-XhoI & ACAGCTCGAGGATAACTAATTTTGGAGAA & BiFC vector cloning \\
\hline VIGS-LTSF1UT-1F & CGACGACAAGACCCTAGGTGTTATTTTTGTCCTTTCC & TRV2 vector cloning \\
\hline VIGS-LTSF1UT-1R & GAGGAGAAGAGCCCTCTGCAAACTCACTGTACAATTTAG & TRV2 vector cloning \\
\hline VIGS-LTSF2UT-1F & CGACGACAAGACCCTGAAAAATGATTTTATGATATC & TRV2 vector cloning \\
\hline VIGS-LTSF2UT-1R & GAGGAGAAGAGCCCTCAATGTATAATTTAGTCCACTGAAAAT & TRV2 vector cloning \\
\hline VIGS-LTSF1/2-1F & CGACGACAAGACCCTTCCAAAACTTGGGAG & TRV2 vector cloning \\
\hline VIGS-LTSF1/2-1R & GAGGAGAAGAGCCCTGCGGTTTCTCTTGTTAAGGGGT & TRV2 vector cloning \\
\hline TRV2:LIC-F & TGTTACTCAAGGAAGCACGATGAGCT & LIC cloning confirmation \\
\hline TRV2:LIC-R & CAGGCACGGATCTACTTAAAGAACGTAG & LIC cloning confirmation \\
\hline LTSF1/2.Spe-F & GCTCACTAGTATGCCTGTCAAAGTAGCA & \\
\hline LTSF1.Asc-R & AATATTGGCGCGCCAGATAACTAATTTTGGAGAA & pMDC83 vector \\
\hline LTSF2.Asc-R & AATATTGGCGCGCCAAATAACTAATTTTGGAGAA & cloning/Transgene \\
\hline Hpt-F & CCTGAACTCACCGCGACG & confirmation \\
\hline Hpt-R & AAGACCAATGCGGAGCATAT & \\
\hline
\end{tabular}

\subsection{Subcellular Localization of the LTSF1 and LTSF2 Proteins}

The LTSF1 coding sequence was amplified in a total volume of $50 \mu \mathrm{L}$ using PrimeStar GXL DNA polymerase (Takara Bio, Kusatsu, Japan) with four-fold-diluted cDNA and 10 pmol of each gene-specific primer (Table 1). The PCR cycling conditions were as follows: initial denaturation at $95^{\circ} \mathrm{C}$ for $3 \mathrm{~min}$; followed by 34 cycles of denaturation at $98{ }^{\circ} \mathrm{C}$ for $10 \mathrm{~s}$, annealing at $60{ }^{\circ} \mathrm{C}$ for $15 \mathrm{~s}$, and extension at $68^{\circ} \mathrm{C}$ for $1 \mathrm{~min}$; with a final extension at $68^{\circ} \mathrm{C}$ for $5 \mathrm{~min}$. The LTSF1 and LTSF2 coding sequences, without the stop codon, were independently inserted into the pMDC83 expression vector at the SpeI-AscI restriction sites using gene-specific primer pairs (Table 1), resulting in the fusion of green fluorescent protein (GFP) to the C-termini of LTSF1 and LTSF2. The recombinant GFP constructs were agro-infiltrated into $N$. benthamiana leaves. Cells harboring an empty pMDC83-GFP vector were used as a control. At $48 \mathrm{~h}$ after agro-infiltration, the GFP signals were detected using a fluorescence microscope (Axioskop 2; Carl Zeiss, Oberkochen, Germany).

\section{5. $\mathrm{Y} 2 \mathrm{H}$ Assays}

The coding sequences of LTSF1, LTSF2, and SKP1 were cloned in frame into the EcoRI-BamHI sites in the pGADT7 and pGBKT7 vectors to generate preys and baits, respectively, using the primers listed in Table 1. The paired constructs were co-transformed into the yeast strain AH109 and grown on a -Leu/-Trp selection medium at $28^{\circ} \mathrm{C}$ for $2-5$ days. Positive yeast transformants were grown on 
SD/-Trp/-Leu/-His/3-AT selection medium for the interaction studies. The GUS assay was performed as described in the Yeast Protocols Handbook.

\subsection{Bimolecular Fluorescence Complementation (BiFC) Analysis}

BiFC analyses were conducted as described by Walter et al. [49]. For the BiFC vector constructs, SKP1, LTSF1, and LTSF2 cDNAs lacking the termination codons were amplified using gene-specific PCR primers (listed in Table 1) and cloned into the EcoRI-BamHI sites of the binary vectors PSPYNE and pSPYCE, which harbor sequences encoding the $\mathrm{YFP}^{\mathrm{N}}$ and $\mathrm{YFP}^{\mathrm{C}}$ (YFP N- and C-termini) protein fragments, respectively. The resulting constructs were named SKP1-YFP ${ }^{\mathrm{N}}$, SKP1-YFPC, LTSF1-YFPN ${ }^{\mathrm{N}}$, LTSF1-YFPC, LTSF2-YFPN , and LTSF2-YFPC. The designated plasmid combinations were co-bombarded into onion (Allium cepa) epidermal cells using the Bio-Rad PDS 1000/He Biolistic Delivery System (Bio-Rad Laboratories, Hercules, CA, USA). On days 1 to 3 after transformation, the onion epidermal cells were visualized using an LSM5 Exciter confocal laser-scanning microscope (LSM 510; Carl Zeiss, Jena, Germany) at NICEM. YFP was excited using a $514 \mathrm{~nm}$ laser beam, and the emissions were detected at 525-600 $\mathrm{nm}$. The BiFC experiment was repeated twice with six biological replicates in each experiment.

\subsection{Genomic DNA Extraction}

Genomic DNA was extracted from young N. benthamiana leaves using the cetyltrimethylammonium bromide method [48]. The concentration and quality of genomic DNA were analyzed using a Nanodrop spectrophotometer (BioTek, Winooski, VT, USA).

\subsection{Agrobacterium-Mediated Transformation of N. benthamiana}

The $p M D C 83-L T S F 1: G F P$ vector constructed above was used for the Agrobacterium-mediated transformation of $N$. benthamiana, which was performed following the method described by Benvenuto et al. [50]. Putative transformants were confirmed using RT-PCR analyses with LTSF1 and Hpt (hygromycin phosphotransferase) gene sequence-specific primers (Table 1). Total RNA was extracted from young $N$. benthamiana leaf samples and cDNA was synthesized as described below. RT-PCR was performed in a total volume of $25 \mu \mathrm{L}$ containing $4 \mu \mathrm{L}$ diluted cDNA template, $2.5 \mu \mathrm{L} 10 \times$ PCR buffer, $2.0 \mu \mathrm{L} 2.5 \mathrm{mM}$ dNTP mix, $0.5 \mu \mathrm{L}$ each of $10 \mu \mathrm{M}$ forward and reverse primers, $0.25 \mu \mathrm{L}$ homemade Taq polymerase, and $15.25 \mu \mathrm{L}$ of water. The PCR cycling conditions were as follows: initial denaturation at $94{ }^{\circ} \mathrm{C}$ for $5 \mathrm{~min}$; followed by 32 cycles of denaturation at $94{ }^{\circ} \mathrm{C}$ for $60 \mathrm{~s}$, annealing at $55^{\circ} \mathrm{C}$ for $60 \mathrm{~s}$, and extension at $72{ }^{\circ} \mathrm{C}$ for $60-90 \mathrm{~s}$; with a final extension at $72{ }^{\circ} \mathrm{C}$ for $5 \mathrm{~min}$. The identified $\mathrm{T}_{0}$ plants were micro-propagated using Murashige and Skoog [51] media to obtain multiple plants for the subsequent analysis.

\subsection{Low-Temperature Stress Treatments}

Approximately two-week-old transgenic and vector control (VC) N. benthamiana plants with a uniform appearance were grown in a walk-in growth chamber at $14{ }^{\circ} \mathrm{C}$ and $25^{\circ} \mathrm{C}$. Five plants per treatment were used in the stress experiments. After one month, the plant heights were recorded and leaf samples were collected for the qPCR analysis.

\subsection{Total RNA Extraction and Reverse-Transcription Quantitative PCR (RT-qPCR)}

To analyze the expression of LTSF1 and LTSF2, systemic leaves were collected from the VIGS pepper plants. The confirmation of the expression of the transgenes and antioxidant enzyme-encoding genes in the N. benthamiana plants were also performed using leaf tissues. Total RNA was extracted from these samples using an MG RNAzol kit (MGmed, Seoul, Korea), according to the manufacturer's instructions. The concentration and integrity of RNA were verified using $1 \%$ agarose gel electrophoresis and a Nanodrop spectrophotometer (BioTek, Winooski, VT, USA). A 1- $\mu$ g aliquot of total RNA was 
used to produce cDNA using an EasyScript Reverse Transcriptase kit (TransGen Biotech, Beijing, China), according to the manufacturer's instructions. The resulting cDNAs were used for further expression studies.

The qPCR analysis was performed using the primers listed in Table S2. The expression levels of genes encoding the antioxidant enzymes were analyzed using a Light Cycler 2.0 instrument (Roche, Basel, Switzerland) using the following conditions: $95^{\circ} \mathrm{C}$ for $4 \mathrm{~min}$; followed by 42 cycles of $95^{\circ} \mathrm{C}$ for $10 \mathrm{~s}, 58^{\circ} \mathrm{C}$ for $20 \mathrm{~s}$, and $72{ }^{\circ} \mathrm{C}$ for $20 \mathrm{~s}$. Three biological replicates and three technical replicates were used for each gene. The relative expression levels were calculated by normalizing the expression levels to the endogenous reference gene Actin. The experiment was repeated twice.

\subsection{Statistical Analysis}

The statistical significance of the data was tested using Student's t-test, and the analyses were performed using Microsoft Excel 2016 (Microsoft, Redmond, WA, USA).

\section{Conclusions}

In conclusion, to elucidate the role of F-box genes in regulating the low-temperature sensitivity of pepper, we knocked down the expression of LTSF1 and LTSF2 genes using VIGS. The pepper plants lacking transcripts for both of these F-box genes exhibited an abnormal phenotype under low-temperature conditions, suggesting that they play an important function in plant adaptation to low-temperature stress. Furthermore, LTSF1 and LTSF2 function as part of the SCF complex, and could, therefore, modulate the degradation of the cellular regulatory proteins and enhance the plant response to low-temperature stress. Transgenic N. benthamiana plants overexpressing LTSF1 showed less growth inhibition and the enhanced expression of genes encoding ROS-scavenging enzymes. The further functional analysis of the LTSF1 and LTSF2 genes and the identification of their novel protein-protein interaction partners and regulatory sites will be essential for the elucidation of their precise functional consequences.

Supplementary Materials: The following are available online at http://www.mdpi.com/2223-7747/9/9/1186/s1, Figure S1. Multiple sequence alignment of the LTSF1 and LTSF2 proteins from 'No.3341' and 'sy-2'. Figure S2. RT-PCR analysis of N. benthamiana transgenic plants. TG-1 to TG5: LTSF1 N. benthamiana transgenic plants; Hpt, hygromycin phosphotransferase gene; M, molecular weight marker; WT, wild type N. benthamiana plant, $\mathrm{Ve}+$, pMDC83-LTSF1:GFP vector. Figure S3. Subcellular localization of the LTSF1 and LTSF2 proteins. In N. benthamiana leaves transiently expressing 35S::LTSF1-GFP or 35S::LTSF2-GFP, the GFP signal was observed in the nucleus, cytoplasm, and cell membrane. pMDC83, empty GFP vector. Table S1. LTSF1 and LTSF2 cDNA sequences. Table S2. qPCR primers used in this study. Reference [52] is cited in supplementary Table S2.

Author Contributions: B.-C.K. and J.V. designed and conceived the research. J.V. developed the transgenic lines. L.L. and J.V. performed the VIGS experiments; J.V., M.-Y.K., and J.-K.K. performed the BiFC and yeast Y2H assays. J.V., M.-Y.K., and J.-K.K. performed the other experiments. J.V. wrote the paper. J.V. and B.-C.K. revised the manuscript. All authors have read and agreed to the published version of the manuscript.

Funding: This work was carried out with the support of the Cooperative Research Program for Agriculture Science \& Technology Development (Project No. PJ01327801), Rural Development Administration (RDA), and Golden Seed Project (Project No. 213006-05-4-SB540), Ministry of Agriculture, Food and Rural affairs (MOF), Rural Development Administration (RDA), and Korea Forest service (KFS), Republic Korea.

Conflicts of Interest: The authors declare no conflict of interest.

\section{References}

1. Chinnusamy, V.; Zhu, J.K.; Sunkar, R. Gene regulation during cold stress acclimation in plants. Methods Mol. Biol. 2010, 639, 39-55. [PubMed]

2. Venkatesh, J.; Kang, B.C. Current views on temperature-modulated R gene-mediated plant defense responses and tradeoffs between plant growth and immunity. Curr. Opin. Plant Biol. 2019, 50, 9-17. [CrossRef] [PubMed] 
3. Korres, N.E.; Norsworthy, J.K.; Tehranchian, P.; Gitsopoulos, T.K.; Loka, D.A.; Oosterhuis, D.M.; Gealy, D.R.; Moss, S.R.; Burgos, N.R.; Miller, M.R.; et al. Cultivars to face climate change effects on crops and weeds: A review. Agr. Sust. Devel. 2016, 36, 12. [CrossRef]

4. Thomashow, M.F. Plant cold acclimation: Freezing tolerance genes and regulatory mechanisms. Annu. Rev. Plant Physiol. 1999, 50, 571-599. [CrossRef] [PubMed]

5. Van Heerden, P.D.; Strasser, R.J.; Kruger, G.H. Reduction of dark chilling stress in N-fixing soybean by nitrate as indicated by chlorophyll a fluorescence kinetics. Physiol. Plant. 2004, 121, 239-249. [CrossRef]

6. An, S.J.; Pandeya, D.; Park, S.W.; Li, J.J.; Kwon, J.K.; Koeda, S.; Hosokawa, M.; Paek, N.C.; Choi, D.; Kang, B.C. Characterization and genetic analysis of a low-temperature-sensitive mutant, sy-2, in Capsicum chinense. Theor. Appl. Genet. 2011, 122, 459-470. [CrossRef]

7. Koeda, S.; Hosokawa, M.; Kang, B.C.; Tanaka, C.; Choi, D.; Sano, S.; Shiina, T.; Doi, M.; Yazawa, S. Defense response of a pepper cultivar cv. Sy-2 is induced at temperatures below $24{ }^{\circ}$ C. J. Plant Res. 2012, 125, 137-145.

8. Koeda, S.; Hosokawa, M.; Saito, H.; Doi, M. Temperature-sensitive phenotype caused by natural mutation in Capsicum latescent in two tropical regions. J. Plant Res. 2013, 126, 675-684. [CrossRef]

9. Almadanim, M.C.; Alexandre, B.M.; Rosa, M.T.; Sapeta, H.; Leitão, A.E.; Ramalho, J.C.; Lam, T.T.; Negrão, S.; Abreu, I.A.; Oliveira, M.M. Rice calcium-dependent protein kinase OsCPK17 targets plasma membrane intrinsic protein and sucrose-phosphate synthase and is required for a proper cold stress response. Plant Cell Environ. 2017, 40, 1197-1213. [CrossRef]

10. Domon, J.M.; Baldwin, L.; Acket, S.; Caudeville, E.; Arnoult, S.; Zub, H.; Gillet, F.; Lejeune-Hénaut, I.; Brancourt-Hulmel, M.; Pelloux, J.; et al. Cell wall compositional modifications of Miscanthus ecotypes in response to cold acclimation. Phytochemistry 2013, 85, 51-61. [CrossRef]

11. Kielbowicz-Matuk, A.; Rey, P.; Rorat, T. The organ-dependent abundance of a Solanum lipid transfer protein is up-regulated upon osmotic constraints and associated with cold acclimation ability. J. Exp. Bot. 2008, 59, 2191-2203. [CrossRef] [PubMed]

12. Orvar, B.L.; Sangwan, V.; Omann, F.; Dhindsa, R.S. Early steps in cold sensing by plant cells: The role of actin cytoskeleton and membrane fluidity. Plant J. 2000, 23, 785-794. [CrossRef] [PubMed]

13. Miquel, M.F.; Browse, J.A. High-oleate oilseeds fail to develop at low temperature. Plant Physiol. 1994, 106, 421-427. [CrossRef] [PubMed]

14. Zhang, J.; Liu, H.; Sun, J.; Li, B.; Zhu, Q.; Chen, S.; Zhang, H. Arabidopsis Fatty Acid Desaturase FAD2 is required for salt tolerance during seed germination and early seedling growth. PLoS ONE 2012, 7, e30355. [CrossRef]

15. Havaux, M.; Kloppstech, K. The protective functions of carotenoid and flavonoid pigments against excess visible radiation at chilling temperature investigated in Arabidopsis npq and tt mutants. Planta 2001, 213, 953-966. [CrossRef]

16. Hua, J.; Grisafi, P.; Cheng, S.H.; Fink, G.R. Plant growth homeostasis is controlled by the Arabidopsis BON1 and BAP1 genes. Genes Dev. 2001, 15, 2263-2272. [CrossRef]

17. Millerd, A.; McWilliam, J.R. Studies on a maize mutant sensitive to low temperature I. Influence of temperature and light on the production of chloroplast pigments. Plant Physiol. 1968, 43, 1967-1972. [CrossRef]

18. Peng, Y.; Zhang, Y.; Lv, J.; Zhang, J.; Li, P.; Shi, X.; Wang, Y.; Zhang, H.; He, Z.; Teng, S. Characterization and fine mapping of a novel rice albino mutant low temperature albino 1. J. Genet. Genom. 2012, 39, 385-396. [CrossRef]

19. Liu, L.; Venkatesh, J.; Jo, Y.D.; Koeda, S.; Hosokawa, M.; Kang, J.H.; Goritschnig, S.; Kang, B.C. Fine mapping and identification of candidate genes for the $s y$-2 locus in a temperature-sensitive chili pepper (Capsicum chinense). Theor. Appl. Genet. 2016, 4, 1-6. [CrossRef]

20. Smalle, J.; Vierstra, R.D. The ubiquitin 26 S proteasome proteolytic pathway. Annu. Rev. Plant Biol. 2004, 55, 555-590. [CrossRef]

21. Lechner, E.; Achard, P.; Vansiri, A.; Potuschak, T.; Genschik, P. F-box proteins everywhere. Curr. Opin. Plant Biol. 2006, 9, 631-638. [CrossRef] [PubMed]

22. Xu, G.; Ma, H.; Nei, M.; Kong, H. Evolution of F-box genes in plants: Different modes of sequence divergence and their relationships. Proc. Natl. Acad. Sci. USA 2009, 106, 835-840. [CrossRef] [PubMed]

23. Yang, X.; Kalluri, U.C.; Jawdy, S.; Gunter, L.E.; Yin, T.; Tschaplinski, T.J.; Weston, D.J.; Ranjan, P.; Tuskan, G.A. The F-box gene family is expanded in herbaceous annual plants relative to woody perennial plants. Plant Physiol. 2008, 148, 1189-1200. [CrossRef] [PubMed] 
24. Gagne, J.M.; Downes, B.P.; Shiu, S.H.; Durski, A.M.; Vierstra, R.D. The F-box subunit of the SCF E3 complex is encoded by a diverse super family of genes in Arabidopsis. Proc. Natl. Acad. Sci. USA 2002, 99, 11519-11524. [CrossRef]

25. Jain, M.; Nijhawan, A.; Arora, R.; Agarwal, P.; Ray, S.; Sharma, P.; Kapoor, S.; Tyagi, A.K.; Khurana, J.P. F-box proteins in rice. Genome-wide analysis, classification, temporal and spatial gene expression during panicle and seed development, and regulation by light and abiotic stress. Plant Physiol. 2007, 143, 1467-1483. [CrossRef]

26. Stefanowicz, K.; Lannoo, N.; Van Damme, E.J. Plant F-box proteins-judges between life and death. Crit. Rev. Plant Sci. 2015, 34, 523-552. [CrossRef]

27. Chae, E.; Tan, Q.K.; Hill, T.A.; Irish, V.F. An Arabidopsis F-box protein acts as a transcriptional co-factor to regulate floral development. Development 2008, 135, 1235-1245. [CrossRef]

28. Chen, Y.; Xu, Y.Y.; Luo, W.; Li, W.X.; Chen, N.; Zhang, D.J.; Chong, K. The F-Box protein OsFBK12 targets OsSAMS1 for degradation and affects pleiotropic phenotypes, including leaf senescence, in rice. Plant Physiol. 2013, 163, 1673-1685. [CrossRef]

29. Potuschak, T.; Lechner, E.; Parmentier, Y.; Yanagisawa, S.; Grava, S.; Koncz, C.; Genschik, P. EIN3-dependent regulation of plant ethylene hormone signaling by two Arabidopsis F box proteins: EBF1 and EBF2. Cell 2003, 115, 679-689. [CrossRef]

30. Sonnberg, S.; Fleming, S.B.; Mercer, A.A. A truncated two-alpha-helix F-box present in poxvirus ankyrin-repeat proteins is sufficient for binding the SCF1 ubiquitin ligase complex. J. Gen. Virol. 2009, 90, 1224-1228. [CrossRef]

31. Song, J.B.; Wang, Y.X.; Li, H.B.; Li, B.W.; Zhou, Z.S.; Gao, S.; Yang, Z.M. The F-box family genes as key elements in response to salt, heavy mental, and drought stresses in Medicago truncatula. Funct. Integr. Genomic. 2015, 15, 495-507. [CrossRef] [PubMed]

32. Takahara, M.; Magori, S.; Soyano, T.; Okamoto, S.; Yoshida, C.; Yano, K.; Sato, S.; Tabata, S.; Yamaguchi, K.; Shigenobu, S.; et al. Too much love, a novel Kelch repeat-containing F-box protein, functions in the long-distance regulation of the legume-Rhizobium symbiosis. Plant Cell Physiol. 2013, 54, 433-447. [CrossRef] [PubMed]

33. Takase, T.; Nishiyama, Y.; Tanihigashi, H.; Ogura, Y.; Miyazaki, Y.; Yamada, Y.; Kiyosue, T. LOV KELCH PROTEIN2 and ZEITLUPE repress Arabidopsis photoperiodic flowering under non-inductive conditions, dependent on FLAVIN-BINDING KELCH REPEAT F-BOX1. Plant J. 2011, 67, 608-621. [CrossRef]

34. Zhang, X.; Gou, M.; Liu, C.J. Arabidopsis Kelch repeat F-box proteins regulate phenylpropanoid biosynthesis via controlling the turnover of phenylalanine ammonialyase. Plant Cell 2013, 25, 4994-5010. [CrossRef]

35. Rao, V.; Petla, B.P.; Verma, P.; Salvi, P.; Kamble, N.U.; Ghosh, S.; Kaur, H.; Saxena, S.C.; Majee, M. Arabidopsis SKP1-like protein13 (ASK13) positively regulates seed germination and seedling growth under abiotic stress. J. Exp. Bot. 2018, 69, 3899-3915. [CrossRef] [PubMed]

36. Yan, Y.S.; Chen, X.Y.; Yang, K.; Sun, Z.X.; Fu, Y.P.; Zhang, Y.M.; Fang, R.X. Overexpression of an F-box protein gene reduces abiotic stress tolerance and promotes root growth in rice. Mol. Plant 2011, 4, 190-197. [CrossRef]

37. Zhou, S.M.; Kong, X.Z.; Kang, H.H.; Sun, X.D.; Wang, W. The involvement of wheat F-box protein gene TaFBA1 in the oxidative stress tolerance of plants. PLOS ONE 2015, 10, e0122117. [CrossRef]

38. Zhou, S.M.; Wang, S.H.; Lin, C.; Song, Y.Z.; Zheng, X.X.; Song, F.M.; Zhu, C.X. Molecular cloning and functional characterisation of the tomato E3 ubiquitin ligase SlBAH1 gene. Funct. Plant Biol. 2016, 43, 1091-1101. [CrossRef]

39. Bu, Q.; Lv, T.; Shen, H.; Luong, P.; Wang, J.; Wang, Z.; Huang, Z.; Xiao, L.; Engineer, C.; Kim, T.H.; et al. Regulation of drought tolerance by the F-box protein MAX2 in Arabidopsis. Plant Physiol. 2014, 164, 424-439. [CrossRef]

40. Gray, W.M.; del Pozo, J.C.; Walker, L.; Hobbie, L.; Risseeuw, E.; Banks, T.; Crosby, W.L.; Yang, M.; Ma, H.; Estelle, M. Identification of an SCF ubiquitin-ligase complex required for auxin response in Arabidopsis thaliana. Genes Dev. 1999, 13, 1678-1691. [CrossRef]

41. Liu, Y.C.; Wu, Y.R.; Huang, X.H.; Sun, J.; Xie, Q. AtPUB19, a U-Box E3 ubiquitin ligase, negatively regulates abscisic acid and drought responses in Arabidopsis thaliana. Mol. Plant 2011, 4, 938-946. [CrossRef] [PubMed]

42. Chen, R.G.; Guo, W.L.; Yin, Y.X.; Gong, Z.H. Novel F-box protein CaF-box is involved in responses to plant hormones and abiotic stress in pepper (Capsicum annuum L.). Int. J. Mol. Sci. 2014, 15, 2413-2430. [CrossRef] 
43. Zhou, S.; Sun, X.; Yin, S.; Kong, X.; Zhou, S.; Xu, Y.; Luo, Y.; Wang, W. The role of the F-box gene TaFBA1 from wheat (Triticum aestivum L.) in drought tolerance. Plant Physiol. Biochem. 2014, 84, 213-223. [CrossRef] [PubMed]

44. Zhao, Z.; Zhang, G.; Zhou, S.; Ren, Y.; Wang, W. The improvement of salt tolerance in transgenic tobacco by overexpression of wheat F-box gene TaFBA1. Plant Sci. 2017, 259, 71-85. [CrossRef]

45. Larkin, M.A.; Blackshields, G.; Brown, N.P.; Chenna, R.; McGettigan, P.A.; McWilliam, H.; Valentin, F.; Wallace, I.M.; Wilm, A.; Lopez, R.; et al. Clustal W and Clustal X version 2.0. Bioinformatics 2007, 23, 2947-2948. [CrossRef] [PubMed]

46. Mitchell, A.; Chang, H.Y.; Daugherty, L.; Fraser, M.; Hunter, S.; Lopez, R.; McAnulla, C.; McMenamin, C.; Nuka, G.; Pesseat, S.; et al. The InterPro protein families database: The classification resource after 15 years. Nucleic Acids Res. 2015, 43, D213-D221. [CrossRef] [PubMed]

47. Fernandez-Pozo, N.; Rosli, H.G.; Martin, G.B.; Mueller, L.A. The SGN VIGS Tool: User-friendly software to design virus-induced gene silencing (VIGS) constructs for functional genomics. Mol. Plant 2015, 8, 486-488. [CrossRef]

48. Hwang, J.; Lee, S.; Lee, J.H.; Kang, W.H.; Kang, J.H.; Kang, M.Y.; Oh, C.S.; Kang, B.C. Plant translation elongation factor $1 \mathrm{~B} \beta$ facilitates potato virus $\mathrm{x}(\mathrm{PVX})$ infection and interacts with PVX triple gene block protein 1. PLOS ONE 2015, 10, e0128014. [CrossRef]

49. Walter, M.; Chaban, C.; Schütze, K.; Batistic, O.; Weckermann, K.; Näke, C.; Blazevic, D.; Grefen, C.; Schumacher, K.; Oecking, C.; et al. Visualization of protein interactions in living plant cells using bimolecular fluorescence complementation. Plant J. 2004, 40, 428-438. [CrossRef]

50. Benvenuto, E.; Ordàs, R.J.; Tavazza, R.; Ancora, G.; Biocca, S.; Cattaneo, A.; Galeffi, P. 'Phytoantibodies': A general vector for the expression of immunoglobulin domains in transgenic plants. Plant Mol. Biol. 1991, 17, 865-874. [CrossRef]

51. Murashige, T.; Skoog, F. A revised medium for rapid growth and bio assays with tobacco tissue cultures. Physiol. Plant. 1962, 15, 473-497. [CrossRef]

52. Li, Y.; Zhang, L.; Wang, X.; Zhang, W.; Hao, L.; Chu, X.; Guo, X. Cotton GhMPK6a negatively regulates osmotic tolerance and bacterial infection in transgenic Nicotiana benthamiana, and plays a pivotal role in development. FEBS J. 2013, 280, 5128-5144. [CrossRef] [PubMed]

(C) 2020 by the authors. Licensee MDPI, Basel, Switzerland. This article is an open access article distributed under the terms and conditions of the Creative Commons Attribution (CC BY) license (http://creativecommons.org/licenses/by/4.0/). 